\title{
Comparison of pellet acceleration model results to experimentally observed penetration depths
}

\author{
T. Szepesi ${ }^{\mathrm{a},{ }^{*}}$, S. Kálvin ${ }^{\mathrm{a}}$, G. Kocsis ${ }^{\mathrm{a}}$, P.T. Lang ${ }^{\mathrm{b}}$, I. Senichenkov ${ }^{\mathrm{c}}$, ASDEX Upgrade Team ${ }^{\mathrm{b}}$ \\ ${ }^{a}$ KFKI - Research Institute for Particle and Nuclear Physics, EURATOM Association, \\ P.O. Box 49, H-1525 Budapest-114, HUNGARY \\ ${ }^{b}$ Max-Planck-Institut für Plasmaphysik, EURATOM Association, \\ Boltzmannstr. 2, 85748 Garching, Germany \\ ${ }^{c}$ Saint Petersburg State Polytechnical University, Polytehnicheskaya 29, \\ 195251 St. Petersburg, Russia
}

\begin{abstract}
Cryogenic hydrogen isotope fuelling pellets were observed to undergo strong radial acceleration in the confined plasma. The reason for pellet acceleration is believed to originate from drift effects: the ionised part of pellet cloud is affected by the grad-B drift, therefore, the cloud becomes polarised. The $\boldsymbol{E} \times \boldsymbol{B}$ drift then deforms the pellet cloud so that it can no longer follow the original flux bundle - this results in a less efficient shielding on the pellet's HFS region, where the subsequently enhanced ablation pushes the pellet towards LFS, like a rocket. In order to study this effect, a simple and a comprehensive ablation model was developed. Results from both models show quantitatively acceptable agreement with ASDEX-Upgrade experiments concerning trajectory curvature, corresponding to radial acceleration in the range of $10^{4}-10^{7} \mathrm{~m} / \mathrm{s}^{2}$.
\end{abstract}

\section{Introduction}

Acceleration of solid hydrogen isotope pellets was observed in ASDEX Upgrade (AUG) tokamak to point also into the $\mathbf{E} \times \mathbf{B}$ drift direction [1], similarly to the ablated material. This was also confirmed by fast framing camera images, indicating a constant vertical velocity component, whereas the radial acceleration was pronounced [2]. For pellets injected from the HFS of the torus both the drifting of the ablated material and pellet acceleration have a positive effect on the fuelling efficiency - a key issue for future fusion reactors, since inefficient fuelling would lead to unwanted confinement degradation and an additional burden for the pumping system, already critical for ITER [3].

Estimating the material deposition profile, thus the fuelling efficiency, of a pellet is strongly affected by the pellet's trajectory, as the deviation from the theoretical straight injection path and the changing velocity can lead either to a deeper or even to a shallower penetration in terms of flux coordinates. This deviation is determined by pellet injection and plasma parameters, but its magnitude can only be estimated by benchmarked simulations. This paper presents two different simulation codes, based on the same considerations, and results are compared to dedicated AUG measurements.

\footnotetext{
* Corresponding author addresses: szepesi.tamas@gmail.com, MTA KFKI-RMKI, P.O. box 49, H-1525 Budapest-114, Hungary
} 


\section{Experiments}

\subsection{Apparatus}

All the experiments presented in this paper were conducted at AUG, a mid-size tokamak with major radius $1.65 \mathrm{~m}$, minor radius $0.5 \mathrm{~m}$, typical plasma volume $13 \mathrm{~m}^{3}$. The centrifuge injector system [4], capable of delivering pellets in three different sizes $\left(1.6,2.7,4.0 \times 10^{20} \mathrm{D}\right.$ atoms), at several velocities $(240,600,880$ and $1000 \mathrm{~m} / \mathrm{s})$ and at frequencies up to $83 \mathrm{~Hz}$, was used. Several plasma scenarios with pellet injection were selected to benchmark the codes: type-I ELMy H-mode discharges with smallest size pellets and several pellet velocities [5], as well as other types of discharges with lower heating power and/or large pellet size to achieve deep penetration (see Table 1 for details).

\begin{tabular}{lccccc} 
Shot & 20040,41 & 20043 & 20054 & 20113 & 23078 \\
\hline Type & H-mode & H-mode & H-mode & H-mode & H-mode \\
$v_{\text {pel }}[\mathrm{m} / \mathrm{s}]$ & 240 & 600 & 1000 & 240 & 600 \\
$m_{\text {pel }}$ orig. [D atoms] & $1.6 \cdot 10^{20}$ & $1.6 \cdot 10^{20}$ & $1.6 \cdot 10^{20}$ & $4.0 \cdot 10^{20}$ & $2.7 \cdot 10^{20}$ \\
$m_{\text {pel }}$ red. [D atoms $]$ & $0.88 \cdot 10^{20}$ & $0.72 \cdot 10^{20}$ & $0.32 \cdot 10^{20}$ & $2.20 \cdot 10^{20}$ & $1.18 \cdot 10^{20}$ \\
$T_{\text {e,ped }}[\mathrm{eV}]$ & 780 & 780 & 780 & 795 & 226 \\
$T_{\text {e,cent. }}[\mathrm{keV}]$ & 3.37 & 3.37 & 3.37 & 4.08 & 0.65 \\
$n_{\text {e,ped }}\left[10^{19} \mathrm{~m}^{-3}\right]$ & 5.26 & 5.26 & 5.26 & 6.05 & 4.97 \\
$n_{\text {e,cent. }}\left[10^{19} \mathrm{~m}^{-3}\right]$ & 8.92 & 8.92 & 8.92 & 11.3 & 12.4 \\
$B_{\mathrm{t}}[\mathrm{T}]$ & -2.7 & -2.7 & -2.7 & -2.7 & -1.8 \\
\hline
\end{tabular}

Table 1. AUG discharge scenarios used in this study. Temperature and density values are derived from profiles used in the simulations. The negative sign in $B_{\mathrm{t}}$ indicates a clockwise magnetic field vector in the torus when seen from the top, while electrons drift upward.

\subsection{Evaluation}

Pellet trajectories were observed by the AUG fast pellet camera system [2]. Long exposure ( $\sim 40 \mathrm{~ms}$ ) images record the complete pellet trajectory; by assuming that the pellet's motion is two-dimensional (the pellet stays in the poloidal plane of the injection). Multiple camera view experiments have shown that this assumption is a good estimate. Then, the real coordinates $(R, z)$ of the trajectory can be reconstructed from a single camera view by assuming that the observation system can be modelled by a pin-hole arrangement.

\section{Pellet acceleration models}

Most pellet ablation models regard the pellet cloud to have a spherical neutral part and another, ionised part, elongated along the magnetic field lines. Both the neutral and the ionised cloud shield the pellet from the incoming hot electrons. The models in this paper are based on the very same idea: the ionised cloud gets polarised by the grad-B drift, and due to the $\mathbf{E} \times \mathbf{B}$ drift the cloud abandons the original flux bundle (see Figure 1). Therefore, the shielding effect of the plasma cloud will be reduced on the pellet's HFS and increased on its LFS, yielding an ablation asymmetry between the pellet's two sides. The difference between 
the two acceleration models is how this ablation asymmetry is made use of in order to calculate the acceleration of the pellet. In both models, just as in the case of the camera observations, it is assumed that the pellet motion is two-dimensional.

\subsection{Pellet acceleration by cloud pressure asymmetry ('NGS model')}

Although the neutral gas shielding (NGS) model of Parks [6] is radially symmetric, in this acceleration model it is assumed that the ablation on the pellet's HFS side is higher, in order to calculate pellet acceleration. The reason for asymmetry may be that the plasma cloud is affected by drifts, therefore, it provides less shielding on the pellets HFS side, or, if we do not wish to consider the shielding effect of the plasma cloud (to remain consistent with the NGS model), we can assume that the drifting plasma cloud exerts a frictious-like force on the neutral cloud, pushing it towards the LFS, which then similarly causes a lower protection on the pellet's HFS side. The enhanced ablation on the HFS will cause a higher cloud pressure there, while on the LFS ablation rate drops and consequently the pressure also decreases.

The principle of this model is that the difference in the cloud pressure on the pellets LFS and HFS is proportional to $p_{0}$, the pressure of the pellet cloud on the pellet surface in the unperturbed case. The latter can be calculated from bulk plasma and pellet parameters [6]

$$
\begin{gathered}
p_{\mathrm{HFS}}-p_{\mathrm{LFS}}=\varepsilon \cdot p_{0} \\
p_{0}=1.3825 \cdot 10^{-12} \cdot n_{e}^{2 / 3} \cdot T_{e}^{1.54} \cdot r_{p}^{-1 / 3}
\end{gathered}
$$

where $p_{0}$ is the cloud pressure on the pellet surface $\left[\mathrm{N} / \mathrm{cm}^{2}\right], n_{\mathrm{e}}$ and $T_{\mathrm{e}}$ are the ambient plasma density $\left[\mathrm{cm}^{-3}\right]$ and temperature $[\mathrm{eV}]$, and $r_{\mathrm{p}}$ is pellet radius $[\mathrm{cm}]$. Therefore, the acceleration of the pellet is as follows:

$$
a=\frac{\left(p_{\mathrm{HFS}}-p_{\mathrm{LFS}}\right) \cdot r_{p}^{2} \pi}{m_{p}}=\varepsilon \frac{p_{0} \cdot r_{p}^{2} \pi}{m_{p}}
$$

This instantaneous acceleration formula is incorporated into an ablation model which calculates pellet dynamics in a given plasma and pellet injection configuration, as well as pellet mass degradation by ablation, based on the NGS formula

$$
\dot{N}_{N G S}=1.12 \cdot 10^{16} \cdot n_{e}^{1 / 3} \cdot T_{e}^{1.64} \cdot r_{p}^{4 / 3} \cdot M_{i}^{-1 / 3}
$$

where $M_{\mathrm{i}}$ is the pellet particle mass in atomic units. The only external parameter of this model is the pressure asymmetry $(\varepsilon)$.

\subsection{Pellet acceleration by rocket-effect ('rocket model')}

In this subsection we follow Ref. [7], notations correspond to [8]. In this section $\dot{N}$ is dependent on the spherical angles, denoted by $\dot{N}(\theta, \varphi)$. Assume the spherically symmetric neutral cloud and the cigar-like plasma cloud have typical densities, temperatures and 
expansion velocities $n_{\mathrm{N}}, T_{\mathrm{N}}, c_{\mathrm{SN}}$ and $n_{\mathrm{p}}, T_{\mathrm{p}}, c_{\mathrm{sp}}$ correspondingly. In [7] the scaling law expressions are derived for ablation rate and cloud parameters ([7], [8]).

Due to the $\mathbf{E} \times \mathbf{B}$ drift of the plasma cloud its thickness $l_{\mathrm{z}}$ depends on $x$ (the radial coordinate) according to $l_{z}(x)=\sqrt{R\left(l_{i}+x\right)}$, where $R$ is the tokamak major radius (see Figure 1). Consequently the quantities describing the shielding cloud $\left(\beta, n_{\mathrm{N}}, T_{\mathrm{N}}\right)$ are $x$-dependent and $\dot{N}(\theta, \varphi) d \Omega / 4 \pi r_{p}^{2}=n_{N}(\theta, \varphi) c_{s N}(\theta, \varphi)$, i.e. the $\dot{N}$ on the pellet surface depends on spherical angles through $x=r_{p} \sin \theta \cos \varphi$.

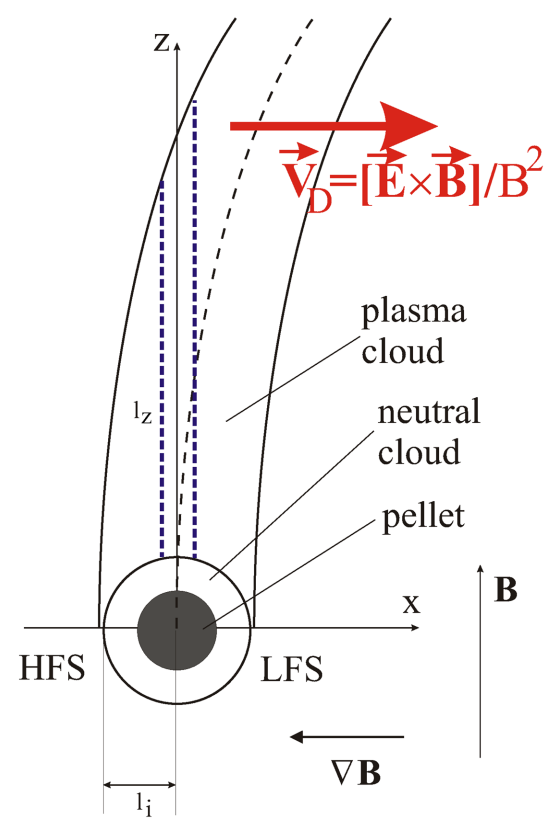

Figure 1. Drifting of the ionised pellet cloud (plasma cloud). The line integrated cloud density (optical thickness, indicated by dashed lines) on the pellet's HFS is reduced compared to the pellet's LFS, therefore ablation is enhanced on the HFS (ablation asymmetry).

Keeping only the first term of Taylor series expansion with regard to small parameter $r_{\mathrm{p}} / l_{\mathrm{i}}$ in equations derived in [7] (see details in [8]), one finds

$$
a=-\frac{3}{4 \pi r_{p}^{2} \rho_{p}} \int \mathrm{d} \Omega \sin \theta \cos \varphi \dot{N}(\theta, \varphi)=-\frac{\sqrt{2 \varepsilon_{\text {vap }} \mu_{I} m_{p}}}{\rho_{p} l_{i} r_{p}} \frac{d \dot{N}}{d x}=-a_{1}-a_{2},
$$

(a sublimation energy of $\varepsilon_{\mathrm{vap}}=0.005 \mathrm{eV}$ is used).

If $\beta<1$ (which is typical for ASDEX-Upgrade parameters) results may be expressed in a form of scalings

$$
a_{1}=\frac{4 \pi}{3 \sqrt[4]{2}} \frac{\sqrt{2 \varepsilon_{v a p} R}}{\rho_{p} \alpha^{5 / 2}}\left(\frac{e^{2}}{4 \pi \varepsilon_{0}}\right)^{\frac{5}{4}}(4 \pi \Lambda)^{\frac{5}{8}} \cdot \sqrt[16]{\frac{(Z(Z+1))^{10} n_{e}^{26} T_{e}^{19} \mu_{I}^{13} m_{p}^{13}}{r_{p}^{14} T_{p}^{5} \cdot E_{i o n}^{26} m_{e}^{13}} \cdot \mathrm{e}^{-\frac{26 e \Delta \Phi}{T_{e}}}}
$$




$$
\begin{gathered}
a_{2}=\frac{4 \pi \alpha^{\frac{7}{2}}}{24} \sqrt[4]{\frac{2}{9}} \frac{\sqrt{2 \varepsilon_{v a p} R}}{\rho_{p}}\left(\frac{e^{2}}{4 \pi \varepsilon_{0}}\right)^{\frac{1}{4}}(4 \pi \Lambda)^{\frac{1}{8}} \cdot \sqrt[16]{\frac{(Z(Z+1))^{2} n_{e}^{18} T_{e}^{23} \mu_{I}^{9} m_{p}^{9}}{r_{p}^{22} T_{p}^{17} \cdot E_{i o n}^{2} m_{e}^{9}} \cdot \mathrm{e}^{-\frac{18 e \Delta \Phi}{T_{e}}}} \cdot \mathrm{e}^{-\sqrt{\frac{l_{z}}{\lambda_{m f p}}}} \\
\frac{l_{z}}{\lambda_{m f p}}=\sqrt[4]{2}(4 \pi \Lambda)^{\frac{7}{8}} \cdot\left(\frac{e^{2}}{4 \pi \varepsilon_{0}}\right)^{\frac{7}{4}} \cdot \sqrt{\alpha R} \cdot \sqrt[16]{\frac{(Z(Z+1))^{14} r_{p}^{6} n_{e}^{14} \mu_{I}^{7} m_{p}^{7}}{T_{e}^{7} T_{p}^{7} \cdot E_{i o n}^{7} m_{e}^{7}} \cdot \mathrm{e}^{-\frac{14 e \Delta \Phi}{T_{e}}}}
\end{gathered}
$$

where $\Delta \Phi$ is the electrostatic shielding potential $\left(\Delta \Phi=2 T_{\mathrm{e}} / e\right.$ in the simulations). Similarly to the first model, these expressions give an instantaneous acceleration, which is incorporated into ablation NGS-like model based on scaling derived in [7].

\section{Results}

For all the discharges listed in section 2, simulations with both codes were performed for the ideal and also for the reduced pellet mass. 'Reduced pellet mass' stands for the average value of pellet particle content which survives the guiding tube system at AUG. The erosion of pellets is strongly dependent on the injection velocity and original pellet size, see details in [4].

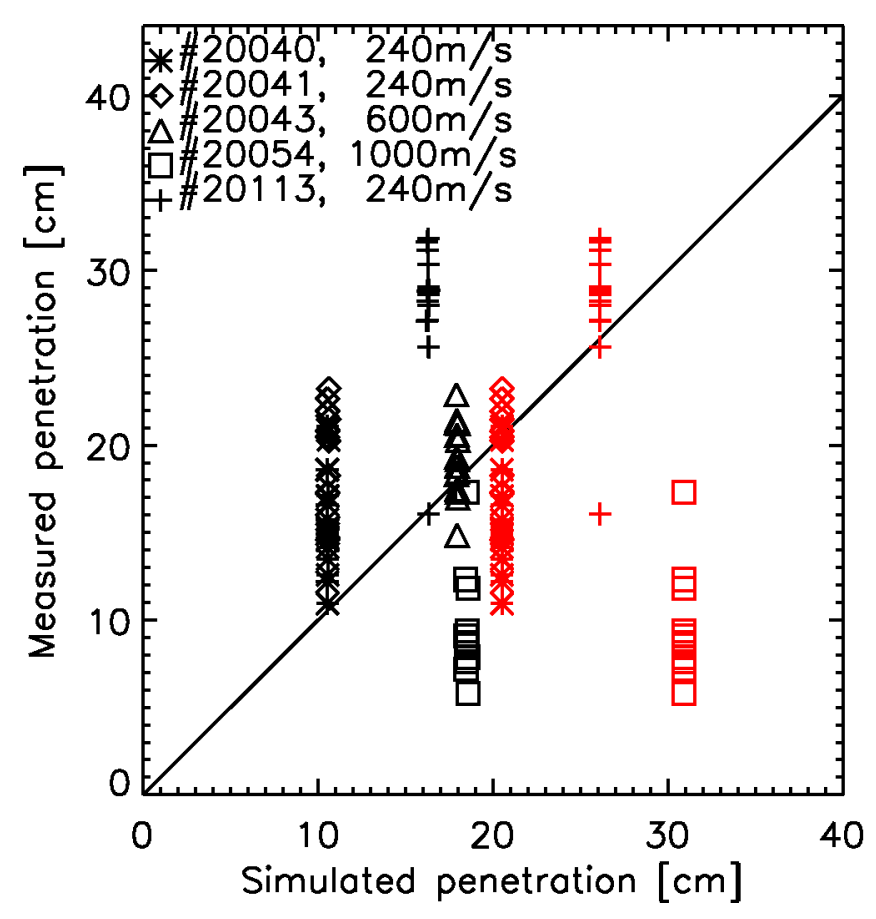

Figure 2. Measured vs. simulated pellet penetration depth. Black symbols: NGS model, grey (red in the online version) symbols: rocket model. To guide the eye an $x=y$ line is overplotted.

The comparison of simulated and measured pellet penetration depths can be seen on Figure 2 . It is obvious that the results of the simulations must be handled with care: despite the large scatter in the measured data, the penetration depth for $240 \mathrm{~m} / \mathrm{s}$ pellets is clearly 
underestimated by the NGS model. On the other hand, the rocket model produces acceptable results (see also Figure 3/a), also for small and large pellet masses (\#20041 and \#20113, respectively). For $600 \mathrm{~m} / \mathrm{s}$ pellets (\#20043, same plasma as in \#20041), the NGS model gives a quantitatively acceptable result; this is also confirmed in a completely different plasma scenario with a significantly lower temperature and magnetic field (Figure $3 / \mathrm{c}$ ). However, for $1000 \mathrm{~m} / \mathrm{s}$ pellets excessively large penetrations are calculated by both models (Figure 3/b).
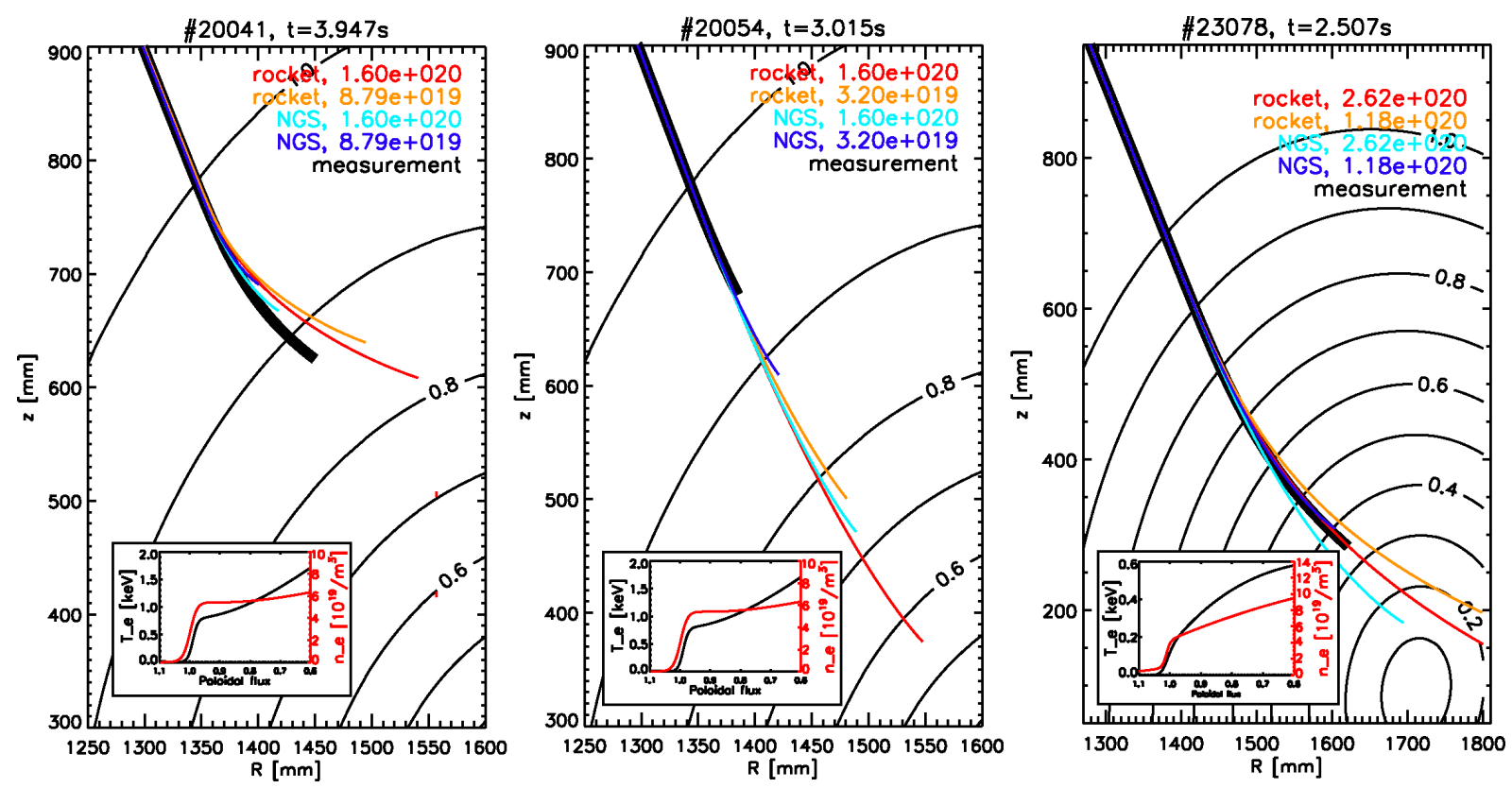

Figure 3. Simulated and measured trajectories for shots \#20041 $\left(v_{\mathrm{pel}}=240 \mathrm{~m} / \mathrm{s}\right), \# 20054\left(v_{\mathrm{pel}}\right.$ $=1000 \mathrm{~m} / \mathrm{s})$ and $\# 23078\left(v_{\text {pel }}=600 \mathrm{~m} / \mathrm{s}, B_{\mathrm{t}}=-1.8 \mathrm{~T}\right)$. The NGS model clearly overestimates the ablation rate, therefore the penetration is smaller than in the measurement, while the rocket model underestimates the ablation, resulting in a significantly deeper penetration. Also, the rocket model overestimates pellet acceleration. However, for $600 \mathrm{~m} / \mathrm{s}$ pellets the NGS code gives a quantitatively good estimate.

Concerning acceleration, the trend is quite the opposite: trajectory shapes for fast pellets are fairly well reproduced (apart from the excess length of the path, see Figure 3), while for 240 $\mathrm{m} / \mathrm{s}$ pellets acceleration is overestimated, resulting in sharply bent trajectories.

\section{Discussion and outlook}

We have to note that in the NGS model the asymmetry value (the cause for acceleration) is an external free parameter, therefore, it can be achieved that the bending of the simulated trajectory would always fit to the measurement by varying the asymmetry parameter accordingly. However, an asymmetry value of 5-7\% was used throughout the whole study. The reason for this was that one could adjust the pellet mass to fit the measured penetration, and then trim the asymmetry to completely fit the simulation result to the measurement. If done so, the asymmetry in all the measured cases was around 5-7\% [9]. Therefore, this value was used also in this study. 
The mismatch of the penetration depths is mainly caused by the shortcomings of the ablation models (and the scaling laws of the ablation rate derived from them), as well as the uncertainty in the temperature profiles - the initial pellet mass has a much weaker effect here. However, the present set of experimental data is not large enough to make stronger statements in this topic.

Although the simulated pellet trajectories were not precisely matching the experimental results, we can say that both models predict the acceleration of pellets in the same order of magnitude. Note that both the comprehensive rocket model and the heuristic NGS model gives about the same trajectory curvature. This - in case the original hypothesis about LFSHFS ablation asymmetry holds - also shows that the strong trajectory curvature is caused by an ablation asymmetry not more than $10 \%$, a value low enough to account for why it could not be seen in the measurements up to now.

Further benchmarking of the codes is planned also at AUG with the new pellet injector ('Blower-gun') being installed in a horizontal LFS injection setup which allows the direct observation of pellet acceleration (in this case deceleration). Also, the models are planned to be compared to JET pellet measurements. The final aim of this benchmarking is to produce a model that would give a quantitatively acceptable result in present machines, and then to predict pellet dynamics in ITER.

\section{References}

[1] H.W. Müller et al., Nucl. Fusion 42 (2002) 301-309.

[2] G. Kocsis et al, Rev. Sci. Instrum. 75 (2004) 4754-4762.

[3] P.T. Lang et al, 11th IAEA Technical Meeting on H-mode Physics and Transport Barriers Tsukuba, Japan, 26-28. September 2007, Pellet perturbations for probing threshold conditions and investigating onset dynamics of paced ELMs (poster)

[4] P.T. Lang et al, Rev. Sci. Instrum 74 (2003) 3974-3983.

[5] G. Kocsis et al, Nucl. Fusion 47 (2007) 1166-1175.

[6] P.B. Parks and R.J. Turnbull, Phys. Fluids 21 (1978) 1735-1741.

[7] V. A. Rozhansky, I. Yu. Senichenkov, Plasma Phys. Reports. 31 (2005) 993.

[8] I. Senichenkov et al., Proceedings of $34^{\text {th }}$ EPS Conference on Plasma Phys., Warsaw, 2007, Europhysics Conference Abstracts Vol. 31F, (European Physical Society), Paper No. P-4.094 (2007).

[9] T. Szepesi et al., Proceedings of $34^{\text {th }}$ EPS Conference on Plasma Phys., Warsaw, 2007. 